\title{
Determination of lunar surface ages from crater frequency-size distribution
}

\author{
B S SHYLAJA \\ Jawaharlal Nehru Planetarium, Bangalore Association for Science Education, \\ High Grounds, Bangalore 560 001, India. \\ e-mail: taralaya@vsnl.com
}

Crater size-frequency distribution is one of the powerful techniques to estimate the ages of planetary surfaces, especially from remote sensing studies. This has been applied to images of the Moon obtained from Clementine mission in 1994. Simple techniques of measurement of the diameter of the craters (in pixels) are used and converted into linear dimensions. Among the several maria studied, the results of Mare Humorum and the central region of Mare Imbrium are reported. The results are compared with age estimates from other sources.

\section{Introduction}

The technique of deriving absolute ages of the lunar surface by using remote sensing techniques was pioneered by Shoemaker and Hackman (1962). Crater counting by itself could be used for obtaining relative ages of the surfaces. The older surfaces accumulate more impacts and hence display a larger number of craters. Thus by knowing the rate of impact, the observed density of craters provides a clue to the age of the surface. The fundamental assumption in the derivation is that the destruction of a crater is slower than its formation. Boyce (1976) carried out extensive studies on crater degradation. The variation in the size distribution of impacting bodies, the variation in the rate of impact, crater degradation, secondary impacts and non-availability of calibration are possible sources of error in this technique. It should be noted that this technique yields model ages only.

The earlier estimates from ground based data and the images from Apollo missions have been calibrated from the lunar soil samples from Apollo and Luna landing sites (Head 1976; Neukum et al 1975). This has extended the application of the technique to other members of the solar system as well (Horedt and Neukum 1984; Stoffler and Ryder 2001). Here we follow the technique described by Heisinger et al $(2000,2003)$ for the data from Clementine. The calibration curve is from Neukum and Ivanov (1994).

\section{The technique}

The crater diameters were measured as precisely as possible from the images by counting the pixels along the equatorial and polar diameters. Clementine provided images of resolution $\sim 1.0 \mathrm{~km} /$ pixel. The regions of Mare Humorum and Mare Imbrium were chosen for this study.

The surface area of the region under consideration was calculated in terms of the pixel size. The crater diameters are sorted into bins of a smaller range. For example craters of diameter 2-8 pixels formed bin 1, 10-19 formed bin $2 \ldots \ldots$ and so on. The cumulative crater frequency $N\left(\mathrm{~km}^{-2}\right)$ for each region was calculated for different diameters of the craters $(1 \mathrm{~km}$ and $10 \mathrm{~km})$ following the lunar chronology curve (Neukum and Ivanov 1994).

The crater diameters are plotted as cumulative distributions, i.e., the number of craters divided by the area of the region covered. This gives a complex continuous curve called the lunar production

Keywords. Craters; ages; lunar basins. 
function. This has been calibrated by various investigators (Hiesinger et al 2000 and references therein). A least square fit is expressed as

$$
\begin{aligned}
N_{\text {cum }}(D>1 \mathrm{~km})= & 5.44 \times 10^{-14}[\exp (6.93 \times t)-1] \\
& +8.38 \times 10^{-4} t
\end{aligned}
$$

where $t$ is the age of the surface in billion years.

This was chosen as the calibration for determining the ages, since it has been verified for the

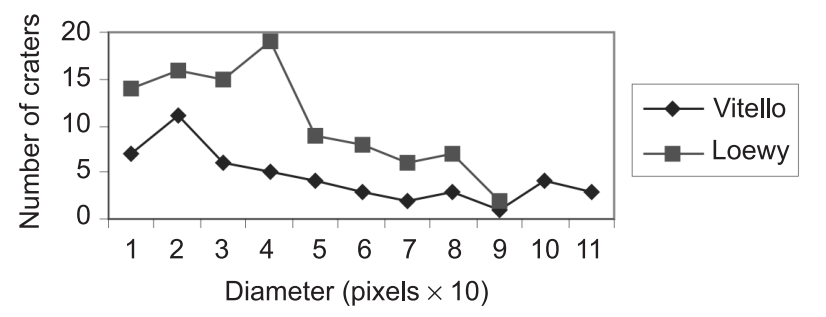

Figure 1. Typical size distribution of craters.

Table 1. The derived model ages of craters.

\begin{tabular}{lc}
\hline Name of the crater & Age (billion year) \\
\hline Vitello & $3.7 \pm 1.3$ \\
Gassendi & $3.6 \pm 0.7$ \\
Flamsteed & $3.7 \pm 1.1$ \\
Kepler & $3.5 \pm 0.8$ \\
Reiner & $3.4 \pm 0.9$ \\
Hansteen & $2.9 \pm 1.2$ \\
Loewy & $2.8 \pm 0.8$ \\
Billy & $3.4 \pm 1.1$ \\
Basin of Imbrium & $3.5 \pm 0.8$ \\
\hline
\end{tabular}

regions of study here (Hiesinger et al 2003). The derivation assumes impact rate and its variation through the geological time scale and calibrates ages against the data from radiometric measurements. The floor basins of craters which were measured for establishing the distribution function were Vitello, Gassendi, Flamsteed, Kepler, Reiner, Hansteen, Loewy and Billy, all on the near side of the Earth. The basin of Imbrium was also included. Figure 1 depicts two examples of the several craters studied.

\section{Discussion}

Earth-based telescopic mapping has been used to estimate the ages of the lunar mare basalts (Pieters et al 1980). The ages of the basins are also derived and classified as Imbrian (2.6-3.92 by), Nectarian (3.98-4.04), pre-Nectarian (ranging from 3.6-4.0). The basin Humorum is Nectarian with an age of about 3.99 billion years. Heisinger et al (2003) puts Copernican as the most recent (up to 1 by BP), followed by Eratosthenian (1.5-3.2 by BP). The oldest are Serentitatis and Imbrium. However, Imbrian age has been regrouped into upper Imbrian (3.22 to about 3.8 by) and lower Imbrian which is a small band of 0.2 billion years due to an extended period of volcanism. Table 1 shows the ages as derived for the craters with errors.

Mare Humorum is believed to be made up of six ring structures of 210,340,425, 570 and $1195 \mathrm{~km}$ diameter (Spudis 1993). The region has been

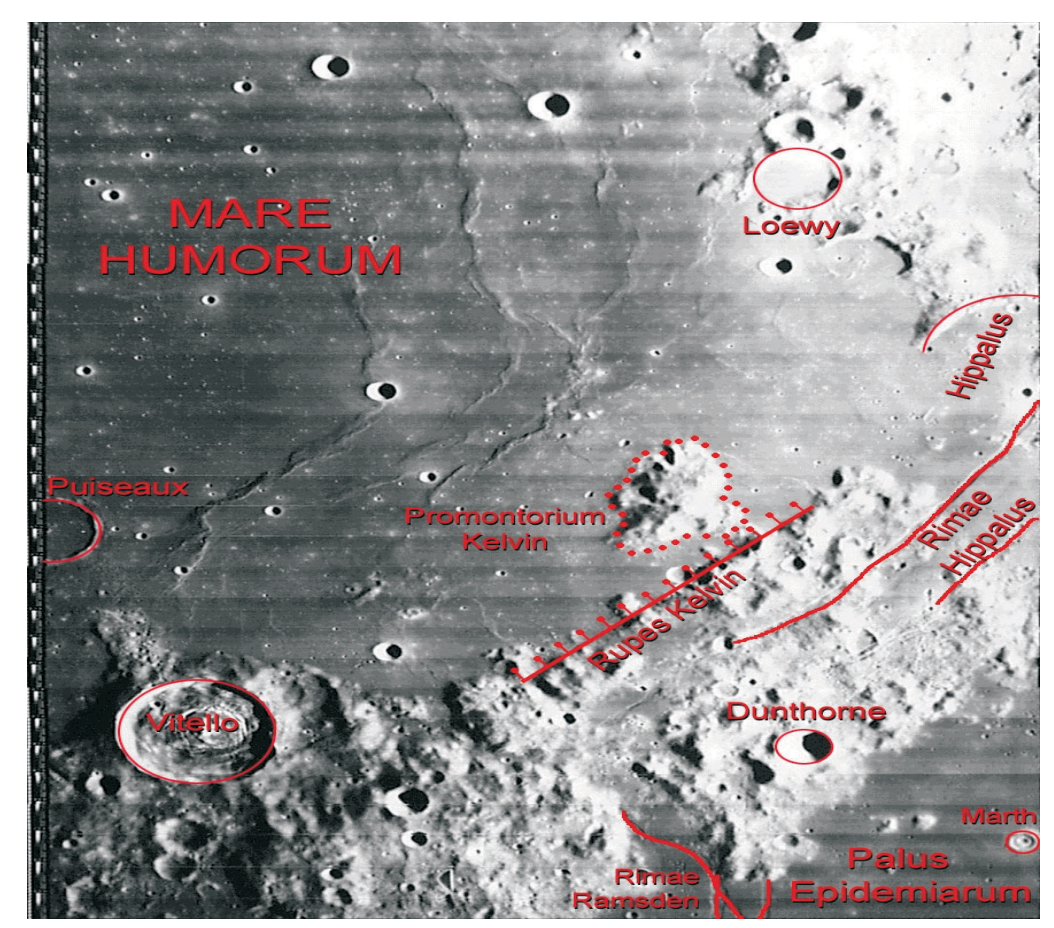

Figure 2. The region of Humorum (from lunar orbiter atlas). 


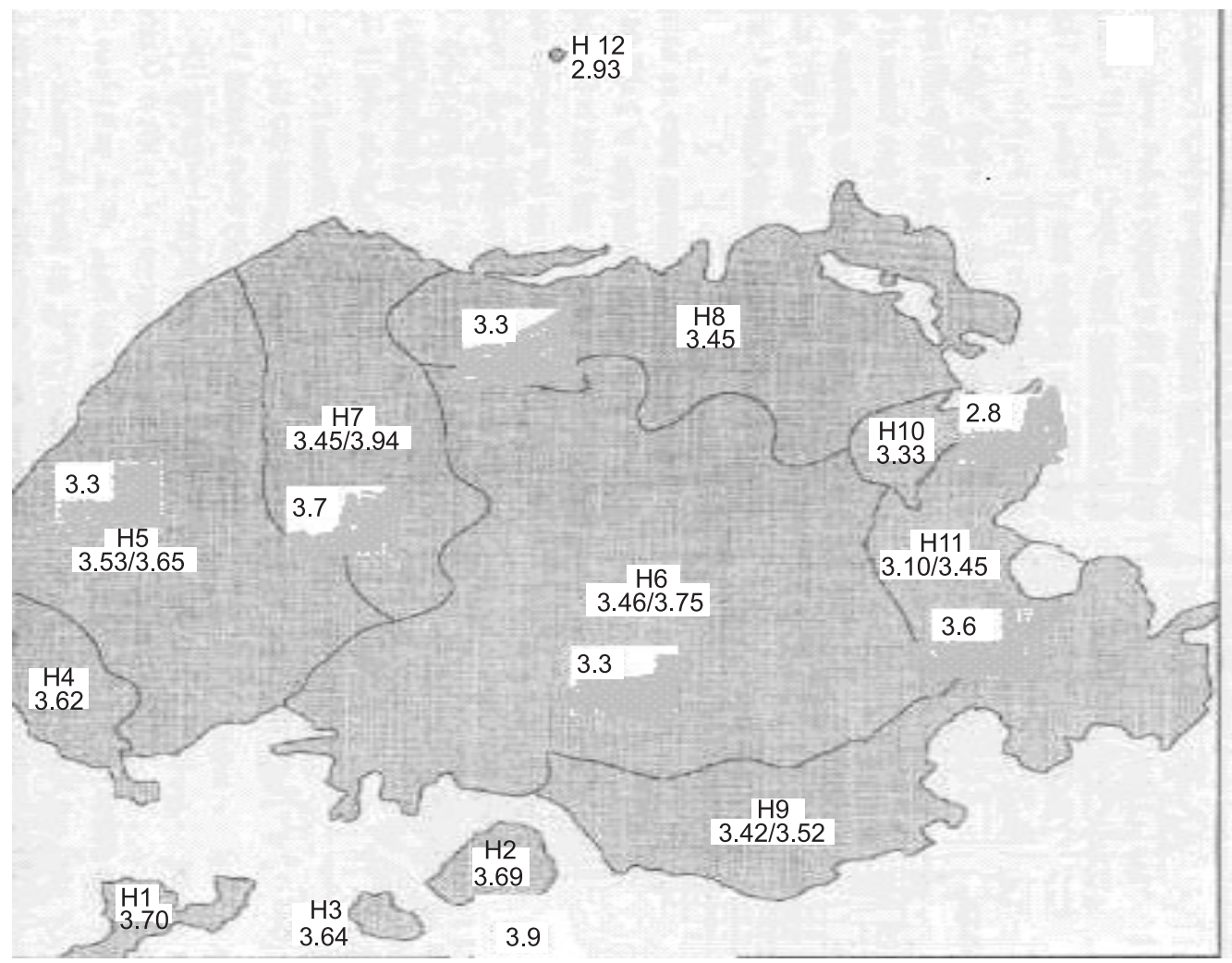

Figure 3. Comparison of ages in the Humorum region with the values of Hiesinger et al (2000).

Table 2. The ages (by) of the regions specified by Hiesinger et al (2000).

\begin{tabular}{clc}
\hline $\begin{array}{c}\text { Region } \\
\text { number }\end{array}$ & $\begin{array}{c}\text { Age (Hiesinger } \\
\text { et al 2000) }\end{array}$ & $\begin{array}{c}\text { Age } \\
\text { (this work) }\end{array}$ \\
\hline H2 & 3.69 & $3.9 \pm 0.8$ \\
H7 & $3.45 / 3.94$ & $3.7 \pm 1.1$ \\
H6 & $3.46 / 3.75$ & $3.2 \pm 1.2$ \\
H8 & 3.45 & $3.3 \pm 0.9$ \\
H10 & 3.33 & $2.8 \pm 0.8$ \\
H11 & $3.10 / 3.45$ & $3.6 \pm 1.0$ \\
\hline
\end{tabular}

further divided into $\mathrm{H} 1, \mathrm{H} 2$, etc., up to $\mathrm{H} 11$ (Heisinger et al 2000) based on colour ratios from multispectral data. The figure 7 in their paper shows the boundaries with the derived ages marked. The ages range over 3.10-3.7 billion years. The sharp change over the boundaries for example from H11 to H6 is striking. This needs to be studied in detail in the context of resurfacing events.

Our results from the Clementine images are tabulated along with the results of Hiesinger et al (2000) based on lunar orbiter images (figure 3). The difference in ages are noticeable in table 2 .

The images of Clementine are considered to be not as good as those from lunar orbiter owing to the unfavourable sun angle. The basic source of error is in the determination of the crater diameter itself. To avoid ambiguity the diameters were measured both in East-West and North-South directions and the mean value has been used. At small dimensions the craters appear as blobs and the edges are not distinct. Thus the determination of the cumulative index could contain a large error. From the calibration curve it may be seen that a small error in this can lead to a larger uncertainty in the age determination especially around 3.0-3.5 billion years.

\section{Conclusions}

The technique of crater diameter measurements is applied to the regions of Mare Humorum. The estimates of ages are consistent with determination from other sources. The unfavourable sun angle and lower resolution results in larger errors.

\section{Acknowledgements}

Acknowledgements are due to NASA for the images from Clementine. This work was done with the help of several undergraduate students K G Geetha, Deepak Dembla, Vinay, Naveen, Jancy Prema, Vijimon, Amar Sharma, Ramakrishna, Harish, Ananth, Ananthakrishna, Ashok and Umesh who very enthusiastically determined the crater diameters. Subsequent analysis was undertaken by the author. I thank Prof. N Bhandari and 
Prof. J N Goswami for financial support. I am grateful to the anonymous referee whose valuable comments have not only improved the presentation but also provided guidelines for future work.

\section{References}

Boyce J M 1976 Ages of flow units in the lunar nearside maria based on Lunar Orbiter IV Photographs; Proc. Lunar. Sci. Conf. 7th, p. 2717.

Head J W III 1976 Lunar Volcanism in space and time; Rev. Geophys. 14265.

Hiesinger H, Jaumann R, Neukum G and Head J W III 2000 Ages of mare basalts on the lunar nearside; J. Geophys. Res. 105 E12, 29,239-29,275.

Hiesinger H, Head J W III, Wolf U, Jaumann R and Neukum G 2003 Ages and Stratigraphy of mare basalts in Oceanus Procellarum, Mare Nubium, Mare Cognitum and Mare Insularum; J. Geophys. Res. 108 1-27.

Horedt G P and Neukum G 1984 Cratering rate over the surface of a synchronous satellite; Icarus 60(3) 710.
Neukum G and Ivanov B A 1994 Crater size distributions and impact probabilities on Earth from lunar, terrestrialplanet and asteroid cratering data; In: Hazard due to Comets and Asteroids (ed.) Gehrels T (Tuscon, Arizona: Univ. Arizona Press) p. 359.

Neukum G, Koenig B and Arkani-Hamed J 1975 A study of lunar impact crater size-distribution; Moon 12 201.

Pieters C M, Head J W III, McCord T B, Adams J B, Zisk S and Whitford-Stark J L 1980 Late high-titanium basalts of the western maria: Geology of the Flamsteed region of Oceanus Procellarum; J. Geophys. Res. 853913.

Shoemaker E M and Hackman R 1962 Stratigraphic basis for a lunar time scale; In: The Moon Symposium 14 of the International Astronomical Union (eds) Kopal Z, Mikhailov Z K (San-Diego California: Academic) 289-300.

Spudis P D 1993 The Geology of Multi ring Impact Basins; Cambridge Planet. Sci. Ser. (New York: Cambridge Univ. Press) 8263.

Stoffler D and Ryder G 2001 Stratigraphy and isotope ages of lunar geologic units: Chronological standard for inner solar system; Space Sci. Rev. 96 9-54. 